\title{
Euglycemic diabetic ketoacidosis: a diagnostic and therapeutic dilemma
}

\author{
Prashanth Rawla', Anantha R Vellipuram², Sathyajit S Bandaru³ and Jeffrey Pradeep Raj \\ 1Department of Internal Medicine, Memorial Hospital of Martinsville and Henry County, Martinsville, Virginia, USA, \\ 2Texas Tech University Health Sciences Center, El Paso, Texas, USA, ${ }^{3}$ Senior Research Associate, Beth Israel Deaconess \\ Medical Center, Harvard Medical School, Boston, Massachusetts, USA, and 'Department of Pharmacology, St John's \\ Medical College, Bangalore, India
}

Correspondence should be addressed to P Rawla

Email

rawlap@gmail.com

\section{Summary}

Euglycemic diabetic ketoacidosis (EDKA) is a clinical triad comprising increased anion gap metabolic acidosis, ketonemia or ketonuria and normal blood glucose levels $<200 \mathrm{mg} / \mathrm{dL}$. This condition is a diagnostic challenge as euglycemia masquerades the underlying diabetic ketoacidosis. Thus, a high clinical suspicion is warranted, and other diagnosis ruled out. Here, we present two patients on regular insulin treatment who were admitted with a diagnosis of EDKA. The first patient had insulin pump failure and the second patient had urinary tract infection and nausea, thereby resulting in starvation. Both of them were aggressively treated with intravenous fluids and insulin drip as per the protocol for the blood glucose levels till the anion gap normalized, and the metabolic acidosis reversed. This case series summarizes, in brief, the etiology, pathophysiology and treatment of EDKA.

\section{Learning points:}

- Euglycemic diabetic ketoacidosis is rare.

- Consider ketosis in patients with DKA even if their serum glucose levels are normal.

- High clinical suspicion is required to diagnose EDKA as normal blood sugar levels masquerade the underlying DKA and cause a diagnostic and therapeutic dilemma.

- Blood pH and blood or urine ketones should be checked in ill patients with diabetes regardless of blood glucose levels.

\section{Background}

Diabetic ketoacidosis (DKA) is defined as a clinical triad comprising metabolic acidosis, hyperglycemia and increased ketone bodies in the blood and urine. Hyperglycemia is usually the hallmark for the diagnosis of DKA (1). However, there is a subset of patients in whom the serum glucose levels are within the normal limits, and this condition is termed as euglycemic DKA (EDKA). This phenomenon was first described by Munro et al. where 37 out of 211 DKA patients had normal sugar levels $(<300 \mathrm{mg} / \mathrm{dL})$ along with a plasma bicarbonate level of $<10 \mathrm{mmol} / \mathrm{L}$ at presentation (2). Later, normoglycemia was redefined as $<250 \mathrm{mg} / \mathrm{dL}$. Thus, EDKA is defined as a triad comprising high anion gap metabolic acidosis with positive serum and urine ketones when serum glycemic levels are $<250 \mathrm{mg} / \mathrm{dL}$ (3). In this case series, we report two patients with type I diabetes mellitus (T1DM) who were diagnosed with EDKA. We believe that this case series would serve as a reminder to all practitioners across the world to consider ketosis in a diabetic patient despite their serum glucose levels being within the normal 
range. This case series summarizes, in brief, the etiology, pathophysiology and treatment of EDKA.

\section{Case presentation 1}

A 21-year-old female with T1DM diagnosed five years back and on an insulin pump for the last two years was admitted with complaints of weakness and inability to eat for the past one day. Patient's insulin pump had stopped working two days before visiting the hospital. There was no history of any fever, nausea, vomiting, diarrhea or other symptoms suggestive of any infective pathology. On examination, the patient had moderate dehydration with loss of skin turgor.

\section{Investigation}

Patient's blood glucose levels were checked, and she was found to be normoglycemic. An arterial blood gas analysis revealed metabolic acidosis and low carbon dioxide values. This was followed by a complete blood work-up that included a hemogram, electrolytes and renal function tests, the results of which along with reference values are given in Table 1 . The patient's urine was positive for ketone bodies with increased ketonemia. There was evidence of dehydration and resulting hemoconcentration along with features suggestive of pre-renal failure. The arterial blood gas (ABG) revealed a partially compensated increased

Table 1 Laboratory investigations of patient 1.

\begin{tabular}{l}
\hline Laboratory tests (units) \\
\hline Random blood sugar $(\mathrm{mg} / \mathrm{dL})$ \\
Hemoglobin (g/dL) \\
White blood cells (cu mm) \\
Platelets (cu mm) \\
Sodium (mmol/L) \\
Potassium (mmol/L) \\
Chloride (mmol/L) \\
Anion gap \\
Blood urea nitrogen (g/dL) \\
Creatinine (mg/dL) \\
Betahydroxylbuterate/ \\
acetoacetate (mmol/L) \\
Carbon dioxide (mmol/L) \\
Arterial blood gas (ABG) \\
PCO $_{2}$ (mm Hg) \\
PO $_{2}$ \\
Bicarbonate \\
${ }_{\mathrm{pH}}$ \\
$\mathrm{U}_{\text {rine ketones }}$ \\
Urine glucose
\end{tabular}

\begin{tabular}{c}
\hline Patient's values \\
\hline 74 \\
16.2 \\
12000 \\
311000 \\
138 \\
2.6 \\
110 \\
22 \\
42 \\
2.19 \\
2.47 \\
6 \\
14 \\
117 \\
6.1 \\
7.11 \\
$2+$ \\
$3+$
\end{tabular}

anion gap metabolic acidosis. Thus, a diagnosis of EDKA was made.

\section{Treatment}

She was treated with $4 \mathrm{~L}$ bolus of IV normal saline and an insulin drip as per the protocol based on her glucose levels. She was also started on dextrose $5 \% 1 \frac{1}{2}$ normal saline. The basic metabolic profile was monitored every $4 \mathrm{~h}$, and serum glucose levels were checked every hour. When her serum carbon dioxide levels were greater than or equal to 18 and her anion gap was less than 12, her insulin drip was switched off, and she was placed on longacting insulin.

\section{Outcome and follow-up}

Patient was discharged to home on long-acting and shortacting insulin and was advised to get her insulin pump fixed on her next appointment with her endocrinologist.

\section{Case presentation 2}

25-year-old female diagnosed with T1DM 10 years back, on regular treatment with insulin glargine at bedtime and insulin aspart at sliding scale as needed before meals, came with complaints of burning while urinating and highgrade intermittent fever of up to $101 \mathrm{~F}$ associated with chills and rigors. She also complained of nausea since last $12 \mathrm{~h}$ and was therefore unable to eat meals adequately. On physical examination at the time of admission, she had mild suprapubic tenderness, and her mucous membranes were dry. There was no renal angle tenderness, and the rest of the physical examination was normal.

\section{Investigation}

A working diagnosis of urinary tract infection was made, and a routine blood work-up was done, the results of which are given in Table 2 . Since clinical dehydration was out of proportion to the symptoms, based on our previous experiences with T1DM patients, we decided to evaluate the patient for DKA, and this revealed the patient to be suffering from concomitant EDKA secondary to urinary tract infection, starving and severe dehydration. The urine analysis confirmed urinary tract infection, and the blood investigations revealed hemoconcentration, prerenal failure, sepsis and partially compensated increased anion gap metabolic acidosis. 
Table 2 Laboratory investigations of patient 2.

\begin{tabular}{l}
\hline Laboratory tests (units) \\
\hline Random blood sugar (mg/dL) \\
Hemoglobin (g/dL) \\
White blood cells (cu mm) \\
Platelets (cu mm) \\
Sodium (mmol/L) \\
Potassium (mmol/L) \\
Chloride (mmol/L) \\
Anion gap \\
Blood urea nitrogen (g/dL) \\
Creatinine (mg/dL) \\
Betahydroxylbuterate/ \\
acetoacetate (mmol/L) \\
Carbon dioxide (mmol/L) \\
Arterial blood gas (ABG) \\
PCO $_{2}$ (mm Hg) \\
PO $_{2}$ \\
Bicarbonate \\
${ }_{\mathrm{p}} \mathrm{H}$ \\
Urine ketones \\
Urine glucose \\
Urine WBC (cells/hpf) \\
Urine leukocyte esterase \\
Urine nitrites
\end{tabular}

\begin{tabular}{|c|c|}
\hline Patient's values & Reference value \\
\hline 97 & $65-100$ \\
\hline 15.4 & $12-15$ \\
\hline 17000 & 4500-11000 \\
\hline 215000 & $140000-440000$ \\
\hline 136 & $135-145$ \\
\hline 3.7 & $3.5-5.0$ \\
\hline 103 & $98-109$ \\
\hline 26 & $1-10$ \\
\hline 34 & $5-25$ \\
\hline 1.79 & $0.70-1.10$ \\
\hline 3.15 & $0.02-0.27$ \\
\hline 7 & $20-30$ \\
\hline 13 & $35-45$ \\
\hline 87 & $75-100$ \\
\hline 6.7 & $22-26$ \\
\hline 7.03 & $7.35-7.45$ \\
\hline $3+$ & 0 \\
\hline $3+$ & 0 \\
\hline $40-80$ & 0 \\
\hline Positive & Negative \\
\hline Positive & Negative \\
\hline
\end{tabular}

In both our patients, other causes of metabolic acidosis were excluded by testing for urine toxicology screen, blood salicylate, acetaminophen, lactic acid and alcohol levels, which were all within the normal limits. There was no known ingestion of toxic substances in these patients. No history of SGLT-2 inhibitors usage in the above patients.

\section{Treatment}

She was treated with 5L of bolus IV normal saline to reverse the dehydration and was started on insulin drip according to the protocol for her blood glucose levels. She was also started on dextrose 5\% $1 / 2$ normal saline IV. She was treated with IV ceftriaxone for her UTI. Her anion gap closed slowly and her acidosis resolved.

\section{Outcome and follow-up}

Patient was started back on her regular insulin regimen with insulin glargine and insulin aspart and was discharged home.

\section{Discussion}

The American Diabetes Association defines DKA as having a combination of hyperglycemia (serum glucose $>250 \mathrm{mg} /$ $\mathrm{dL}$ ), acidosis (arterial $\mathrm{pH}<7.3$ and bicarbonate $<15 \mathrm{mEq} / \mathrm{L}$ ) and ketosis (moderate ketonuria or ketonemia) (1). Glycemic control is achieved in our human body using a balance between the insulin levels and the levels of counter-regulatory hormones like glucagon, growth hormone, glucocorticoids and epinephrine. DKA occurs when there is either a decrease in insulin or when there is an excess of counter-regulatory hormones both of which causes hyperglycemia. Though there is hyperglycemia, the end organs are unable to utilize the available glucose due to the comparative lack of insulin, and this leads to lipolysis thereby leading to excessive production of ketone bodies (4). However, in this case series, we have reported 2 cases where there is DKA but no hyperglycemia.

The underlying mechanism of EDKA is either due to decreased hepatic production of glucose during fasting state or enhanced urinary excretion of glucose induced by an excess of counter-regulatory hormones, the former being the most common reason. Thus, when a diabetic patient is exposed to any triggering factor for DKA and is fasting or starving while continuing the insulin treatment regularly, the liver will be in a state of glycogen depletion, thereby producing a lesser amount of glucose. On the other hand, there will be lipolysis and fatty acid production, which finally leads to excessive ketone body production (3). Some of the common causes of EDKA that have been reported in literature so far are low caloric intake, fasting or starvation (5), pregnancy (6), pancreatitis (7), cocaine intoxication, prolonged vomiting or diarrhea (8), insulin pump use (9) and of late use of SGLT2 inhibitors like empagliflozin, canagliflozin and so forth (10).

Both our patients were type 1 diabetes mellitus patients on insulin therapy. The first patient had a history of failed insulin pump two days before admission and decreased food intake in the past $24 \mathrm{~h}$. Burge et al had reported in their study that short-term fasting is a well-known mechanism of developing euglycemic ketoacidosis when there is insulin deficiency in type I diabetic patients (11). They also subsequently went ahead to describe how dehydration can accelerate the development of DKA during periods of insulin deficiency. Dehydration usually promotes the development of hyperglycemia. However, it is interesting to note its differential role in EDKA. Fasting primarily increases the secretion of counter-regulatory hormones especially the glucagon, which depletes the glycogen stores in the liver. Dehydration acts as a stimulus for further glucagon secretion, which results in lipolysis and ketone body production in the background of decreased glucose production leading to EDKA. During insulin deficiency, dehydration also increases the secretion of other counter- 
regulatory hormones like catecholamines and cortisol, which further worsens EDKA (12). In the case of our second patient, urinary tract infection in conjunction with nausea due to the infection caused a decreased calorie intake and led to ketoacidosis with euglycemia. This is a classic presentation of EDKA.

Diagnosis of EDKA is difficult as it is primarily a diagnosis of exclusion. Other forms of ketoacidosis like starvation ketoacidosis has to be ruled out. Also, other causes of increased anion gap metabolic acidosis like lactic acidosis, increased toxic serum alcohols (methanol, ethylene glycol, etc.), drug toxicity, paraldehyde ingestion and renal failure have to be excluded (8). Once diagnosed, management of EDKA is simple and is almost similar to the management of DKA. The mainstay of treatment involves rapid correction of dehydration using intravenous fluids (13). The second most important step in the management is the use of insulin drip along with a dextrose containing solution until the anion gap, and bicarbonate levels normalize (14). Periodic checking of urine for ketones and arterial blood gas analysis to estimate anion gap are warranted till the values normalize (13).

Here, we presented two patients diagnosed with euglycemic diabetic ketoacidosis both of whom were on regular insulin therapy. Early detection and management are warranted as this condition may else prove fatal. High clinical suspicion is required to diagnose EDKA as normal blood sugar levels masquerade the underlying DKA and cause a diagnostic and therapeutic dilemma. It is best advised that the clinicians are aware of the possible etiological triggers of EDKA in susceptible patients and actively rule out other differentials thereby minimizing the time required for diagnosing EDKA. If diagnosed early and management aggressively with fluids and insulin drip, EDKA may be easily reversed, thus minimizing morbidity and mortality.

\section{Declaration of interest}

The authors declare that there is no conflict of interest that could be perceived as prejudicing the impartiality of the research reported.

\section{Funding}

This research received no specific grant from any funding agency in the public, commercial, or not-for-profit sectors.
Patient consent

Written informed consent has been obtained from the patients for publication of this article.

Author contribution statement

Study design, drafting by $P R$, critical revisions and final approval by $P R$, A R V, S S B and J P R.

\section{References}

1 Nyenwe EA \& Kitabchi AE 2016 The evolution of diabetic ketoacidosis: an update of its etiology, pathogenesis and management. Metabolism 65 507-521. (doi:10.1016/j. metabol.2015.12.007)

2 Munro JF, Campbell IW, McCuish AC \& Duncan LJ 1973 Euglycaemic diabetic ketoacidosis. BMJ 2 578-580. (doi:10.1136/bmj.2.5866.578)

3 Kitabchi AE, Umpierrez GE, Miles JM \& Fisher JN 2009 Hyperglycemic crises in adult patients with diabetes. Diabetes Care 32 1335-1343. (doi:10.2337/dc09-9032)

4 Laffel L 1999 Ketone bodies: a review of physiology, pathophysiology and application of monitoring to diabetes. Diabetes/Metabolism Research and Reviews 15 412-426. (doi:10.1002/(SICI)15207560(199911/12)15:6<412::AID-DMRR72>3.0.CO;2-8)

5 Joseph F, Anderson L, Goenka N \& Vora J 2009 Starvation induced true diabetic euglycemic ketoacidosis in severe depression. Journal of General Internal Medicine 24 129-131. (doi:10.1007/s11606-008-0829-0)

6 Chico M, Levine SN \& Lewis DF 2008 Normoglycemic diabetic ketoacidosis in pregnancy. Journal of Perinatology 28 310-312. (doi:10.1038/sj.jp.7211921)

7 Prater J \& Chaiban J 2015 Euglycemic diabetic ketoacidosis with acute pancreatitis in a patient not known to have diabetes. Endocrine Practice 1 e88-e91. (doi:10.4158/ep14182.cr)

8 Abdin AA, Hamza M, Khan MS \& Ahmed A 2016 Euglycemic diabetic ketoacidosis in a patient with cocaine intoxication. Case Reports in Critical Care 2016 Article ID: 4275651. (doi:10.1155/2016/4275651)

9 Modi A, Agrawal A \& Morgan F 2017 Euglycemic diabetic ketoacidosis. Current Diabetes Reviews 13 315-321. (doi:10.2174/15733 99812666160421121307)

10 Qui H, Novikov A \& Vallon V 2017 Ketosis and diabetic ketoacidosis in response to SGLT2 inhibitors: basic mechanisms and therapeutic perspectives. Diabetes/Metabolism Research and Reviews 33 e2886. (doi:10.1002/dmrr.2886)

11 Burge MR, Hardy KJ \& Schade DS 1993 Short-term fasting is a mechanism for the development of euglycemic ketoacidosis during periods of insulin deficiency. Journal of Clinical Endocrinology and Metabolism 76 1192-1198. (doi:10.1210/jc.76.5.1192)

12 Burge MR, Garcia N, Qualls CR \& Schade DS 2001 Differential effects of fasting and dehydration in the pathogenesis of diabetic ketoacidosis. Metabolism 50 171-177. (doi:10.1053/meta.2001.20194)

13 Gelaye A, Haidar A, Kassab C, Kazmi S \& Sinha P 2016 Severe ketoacidosis associated with canagliflozin (Invokana): a safety concern. Case Reports in Critical Care 2016 Article ID: 1656182. (doi:10.1155/2016/1656182)

14 Rosenstock J \& Ferrannini E 2015 Euglycemic diabetic ketoacidosis: a predictable, detectable, and preventable safety concern with SGLT2 inhibitors. Diabetes Care 38 1638-1642. (doi:10.2337/dc15-1380)

Received in final form 18 July 2017

Accepted 4 August 2017 